\title{
Italian family with two independent mutations: 3358T/A in BRCA1 and 8756delA in BRCA2 genes
}

\author{
Laura Cortesi ${ }^{1}$, Daniela Turchetti ${ }^{1}$, Chiara Bertoni ${ }^{2}$, Tommaso Zanocco-Marani ${ }^{2}$, Marco \\ Vinceti $^{3}$, Chiara Silvestri ${ }^{1}$, Massimo Federico ${ }^{1}$, Vittorio Silingardi ${ }^{1}$ and Sergio Ferrari ${ }^{\star 2}$
}

\begin{abstract}
${ }^{1}$ Dipartimento di Oncologia ed Ematologia, Università di Modena e Reggio Emilia, Via del Pozzo 71, Modena, Italy; ${ }^{2}$ Dipartimento di Scienze Biomediche, Sezione di Chimica Biologica, Università di Modena e Reggio Emilia, Via Campi 287, Modena, Italy; ${ }^{3}$ Dipartimento di Scienze Igienistiche, Microbiologiche e Biostatistiche, Università di Modena e Reggio Emilia, Via Campi 287, Modena, Italy
\end{abstract}

Hereditary breast/ovarian cancer is a well-characterized clinical entity, largely attributed to the inheritance of BRCA1 or BRCA2 mutations. Among general population, the mutation's frequency of these genes is very low; therefore, the identification of two independent mutations in the same family is a rare event. This study reports the presence of two mutations, one in the BRCA1 and the second in the BRCA2 gene in an Italian Caucasian kindred. This family is composed of more than $\mathbf{2 5 0}$ individuals, spanning through five generations, among which endogamy was a common phenomenon. Considering the tumor spectrum, this family is characterized by a high incidence of different types of cancer. In our study, we considered only three out of seven family units for BRCA1 and BRCA2 analysis. In one of the family units, we found independent mutations of both BRCA genes. The BRCA1 mutation on exon $11(3358 \mathrm{~T} \rightarrow \mathrm{A})$ was identified originally in the index case and subsequently in 18 members of this family, whereas the same mutation was not detected in a related family member with male breast cancer. The male breast cancer patient led to the identification, through mutational analysis, of a new BRCA2 mutation (8756delA). This BRCA2 mutation was also found in the male breast cancer patient's daughter. The discovery of the BRCA2 mutation allowed us to alert the patient's daughter who, otherwise, could be falsely reassured since she had a negative BRCA1 test.

European Journal of Human Genetics (2003) 11, 210-214. doi:10.1038/sj.ejhg.5200948

Keywords: BrCa1; BrCa2; ovarian cancer; male breast cancer; mutational analysis

Introduction

The detection of two independent mutations in both the breast/ovarian cancer susceptibility genes, BRCA1 and BRCA2, is rarely described among the members of the same family. Until now, these mutations were found together in Ashkenazi Jewish kindred ${ }^{1-6}$ and in a few cases of non-Jewish families. ${ }^{7-9}$ It has to be pointed out that

*Correspondence: Dr S Ferrari, Dipartimento di Scienze Biomediche, Sezione di Chimica Biologica, Università di Modena e Reggio Emilia, Via Campi 287, Modena, Italy. Tel: 003959 2055400; Fax 003959 2055410; E-mail: ferrari.sergio@unimore.it

Received 23 July 2002; revised 5 December 2002; accepted 12 December 2002 independent BRCA1 and BRCA2 mutations have never been detected before in Italian families.

We described an extended Italian family where two different non-Ashkenazi Jewish mutations, one in the BRCA1 and the second in the BRCA2 gene, are present in independent members of this family. The two mutations described in the present report have never been observed in the same individual. The genetic testing for BRCA1 and BRCA2 was performed by direct automated sequencing (DAS) in 38 members. In this family, we found this BRCA1 mutation in 19 members, six affected and 13 healthy, and a BRCA2 novel mutation only in a male breast cancer patient and in his healthy daughter. 
The identification of these independent BRCA1 and BRCA2 mutations in the same family, represents the first report in a pedigree not belonging to the Ashkenazi Jewish population and could reflect the possibility of a 'de novo' BRCA2 mutation.

\section{Materials and methods Family}

This five-generation family named M019 (Figure 1), originating from the north of Italy, was composed of more than 250 individuals, with a high global incidence of different types of tumors such as thyroid, lung, brain, kidney, liver and corpus uteri, as well as nine ovarian, 12 female breast, four stomach and one male breast cancers. As demonstrated by Ludwig et al, ${ }^{10}$ BRCA $1^{\text {tr } / \text { tr }}$ mouse homozygous mutants, that lose the C-terminal of the protein, develop different types of tumor, particularly, carcinomas (breast, lung, liver, uterus) and lymphomas. Among the family, 38 individuals, six affected and 32 unaffected, were available for BRCA1 and BRCA2 mutational analysis. The spectrum of cancers associated with the mutation in BRCA1 and BRCA2 genes is shown in Table 1.

\section{BRCA1 and BRCA2 mutational analysis}

Blood samples were obtained from members of the family under informed consent and DNA was extracted using standard protocols.

For BRCA1 analysis we used 17 pairs of primers, as described by Friedman et al, ${ }^{11}$ to amplify exons $2,3,5,6,8$, $11,13,16,17,18,19$ and 20, the most frequently mutated, while for BRCA2 analysis, we designed new sets of PCR primers to study exons 7, 9, 10, 11, 20 and 23. Purification of PCR products, direct sequencing and subcloning of fragments with unclear heterozygosity were carried out as already described. ${ }^{12}$

\section{Statistical methods}

Standardized incidence ratios (SIRs) were calculated by determining the ratio of the observed patients compared to the expected number of cases with all the breast and ovarian cancers. The end point for person-years calculation was 31 December 2000 or the date of diagnosis of the sitespecific neoplasm under investigation or, the date of death or emigration when appropriate. The expected number of subjects with cancer was determined by using 5 -year age, sex and calendar-period specific incidence rates estimated for the Modena province population. ${ }^{13}$ As a whole, the incidence of breast and ovarian cancers was considerably higher than expected (Table 2).

\section{Results}

In all, 38 members of this family were analyzed with DAS for BRCA1 and BRCA2 mutations. We found the $3358 \mathrm{~T} \rightarrow \mathrm{A}$

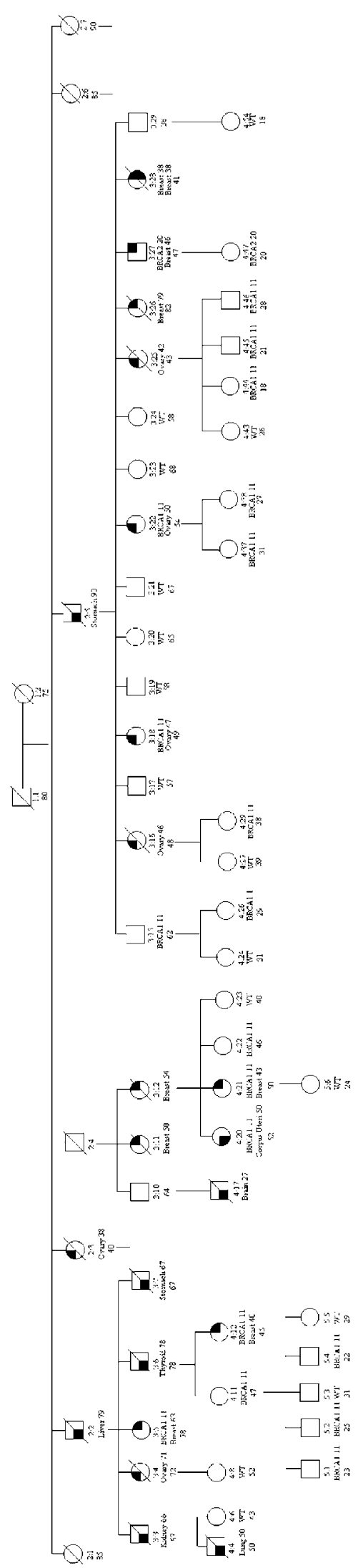


Table 1 Spectrum of cancers associated with the mutations in BRCA1 and BRCA2 genes

\begin{tabular}{|c|c|c|c|c|}
\hline Individual ID & $B R C A 13358 T \rightarrow A$ & BRCA2 8756delA & Type of cancer & Age of onset \\
\hline $1: 1$ & Unknown & Unknown & None & None \\
\hline $1: 2$ & Unknown & Unknown & None & None \\
\hline $2: 2$ & Obligate carrier & Unknown & Liver & 79 \\
\hline $2: 3$ & Unknown & Unknown & Ovary & 38 \\
\hline $2: 4$ & Obligate carrier & Unknown & None & None \\
\hline $2: 5$ & Obligate carrier & Unknown & Stomach & 90 \\
\hline $3: 3$ & Unknown & Unknown & Kydney & 66 \\
\hline $3: 4$ & Unknown & Unknown & Ovary & 71 \\
\hline $3: 5$ & Yes & No & Breast & 63 \\
\hline $3: 6$ & Obligate carrier & No & Thyroid & 78 \\
\hline $3: 7$ & Unknown & Unknown & Stomach & 67 \\
\hline $3: 10$ & Unknown & Unknown & None & None \\
\hline $3: 11$ & Unknown & Unknown & Breast & 50 \\
\hline $3: 12$ & Obligate carrier & No & Breast & 54 \\
\hline $3: 15$ & Yes & No & None & None \\
\hline $3: 16$ & Obliqate carrier & No & Ovary & 46 \\
\hline $3: 17$ & No & No & None & None \\
\hline $3: 18$ & Yes & No & Ovary & 47 \\
\hline $3: 19$ & No & No & None & None \\
\hline $3: 20$ & No & No & None & None \\
\hline $3: 21$ & No & No & None & None \\
\hline $3: 22$ & Yes & No & Ovary & 50 \\
\hline $3: 23$ & No & No & None & None \\
\hline $3: 24$ & No & No & None & None \\
\hline $3: 25$ & Obligate carrier & No & Ovary & 42 \\
\hline $3: 26$ & Unknown & Unknown & Breast & 79 \\
\hline $3: 27$ & No & Yes & Breast & 46 \\
\hline $3: 28$ & Unknown & Unknown & Bilateral breast & 38,38 \\
\hline $3: 29$ & Unknown & Unknown & None & None \\
\hline $4: 4$ & Unknown & Unknown & Lung & 50 \\
\hline $4: 6$ & No & No & None & None \\
\hline $4: 8$ & No & No & None & None \\
\hline $4: 11$ & Yes & No & None & None \\
\hline $4: 12$ & Yes & No & Breast & 40 \\
\hline $4: 17$ & Unknown & Unknown & Brain & 27 \\
\hline $4: 20$ & Yes & No & Uterine & 50 \\
\hline $4: 21$ & Yes & No & Breast & 43 \\
\hline $4: 22$ & Yes & No & None & None \\
\hline $4: 23$ & No & No & None & None \\
\hline $4: 24$ & No & No & None & None \\
\hline $4: 26$ & Yes & No & None & None \\
\hline $4: 27$ & No & No & None & None \\
\hline $4: 29$ & Yes & No & None & None \\
\hline $4: 37$ & Yes & No & None & None \\
\hline $4: 38$ & Yes & No & None & None \\
\hline $4: 43$ & No & No & None & None \\
\hline $4: 44$ & Yes & No & None & None \\
\hline $4: 45$ & Yes & No & None & None \\
\hline $4: 46$ & Yes & No & None & None \\
\hline $4: 47$ & No & Yes & None & None \\
\hline $4: 54$ & No & No & None & None \\
\hline $5: 1$ & Yes & No & None & None \\
\hline $5: 2$ & Yes & No & None & None \\
\hline $5: 3$ & No & No & None & None \\
\hline $5: 4$ & Yes & No & None & None \\
\hline $5: 5$ & No & No & None & None \\
\hline $5: 6$ & No & No & None & None \\
\hline
\end{tabular}

BRCA1 nonsense mutation in three patients with breast cancer, two patients with ovarian cancer and one patient with uterine adenocarcinoma. Furthermore, 13 healthy individuals were carriers of the same BRCA1 mutation.
Since the male breast cancer patient was BRCA1 negative and considering the high incidence of BRCA2 mutations in families with male breast cancer, ${ }^{14}$ we performed mutational analysis for the BRCA2 gene on this patient. As a 
Table 2 Standardized incidence ratio (SIR) of all neoplasms, breast cancer and ovarian cancer in the whole study population

\begin{tabular}{lcccr}
\hline & Observed cases & Expected cases & $S^{2} R^{\mathrm{a}}$ & \multicolumn{1}{c}{$95 \% \mathrm{Cl}$} \\
\hline $\mathrm{AC}^{\mathrm{b}}$ & 22 & 1.37 & 14.6 & $9.4-22.7$ \\
$\mathrm{BC}^{\mathrm{c}}$ & 8 & 0.23 & 25.7 & $11.5-57.1$ \\
$\mathrm{OC}^{\mathrm{d}}$ & 6 & 0.25 & 24.1 & $10.8-53.6$
\end{tabular}

${ }^{\mathrm{a}} \mathrm{SIR}$, standardized incidence ratio. ${ }^{\mathrm{b}} \mathrm{AC}$, all cancers. ${ }^{\mathrm{C}} \mathrm{BC}$, breast cancer. ${ }^{\mathrm{d} O C}$, ovarian cancer.

consequence, we detected a BRCA2 mutation on exon 20, characterized by $8756 \mathrm{delA}$, giving rise to a stop codon insertion at nucleotide 8815 causing a truncated protein product. This mutation had never been identified before and was found also in the patient's healthy daughter. Moreover, all the members tested for BRCA1 analysis were also searched for the 8756delA BRCA2 mutation, but we did not find any additional carrier.

One BRCA1 female breast cancer patient had an in situ ductal carcinoma at 43 years of age (4:21 in Figure 1), whereas two other patients $(3: 5,4: 12)$ were characterized by ductal infiltrating carcinoma with an aggressive phenotype: grading III, hormonal receptors negative, high proliferation rate. ${ }^{12}$ All the four BRCA1 ovarian cancers (3:16, 3:18, 3:22 and 3:25) were serous adenocarcinomas, consistent with the data of other authors. ${ }^{15}$

The male breast cancer (3:27) was a poorly differentiated, infiltrating ductal carcinoma. To evaluate the frequency of this novel deletion in BRCA2 gene, we analyzed 10 other cases of male breast cancer belonging to unrelated families. All the cases studied were negative for this mutation.

Furthermore, in our family where the ratio between ovarian and breast cancers is almost $1: 1$, the results are in accordance with the literature data. The relative risk for breast and ovarian cancer is also similar, being the SIR (see Table 2) 25.7 for $\mathrm{BC}$ and 24.1 for OC. It has to be considered that the high SIR of the other tumors $(\mathrm{AC}=14.6)$ could reflect an involvement of $3358 \mathrm{~T} \rightarrow \mathrm{A}$ BRCA1 mutation in different types of tumors. The high cancer risk in this Italian family is therefore consistent with the studies of Ludwig et $a l^{10}$ and Thompson et al for the Breast Cancer Linkage Consortium. ${ }^{16}$

\section{Discussion}

In this study, we describe an atypical Italian family characterized by a large number of individuals and by endogamy among family members. This family shows two separate mutations segregating with breast and ovarian cancer, among multiple siblings. Our family shares many characteristics with the large kindred of Ashkenazi Jewish, in which BRCA1 and BRCA2 mutations are frequently detected. The 3358T $\rightarrow \mathrm{A}$ BRCA1 mutation is certainly inherited since $50 \%$ of the members of the three family units studied are carriers. Since only the male breast cancer patient and his daughter were found positive for BRCA2 mutational analysis, the most probable interpretation is that this is a 'de novo' mutation. We cannot exclude the possibility that the mutation was inherited from the father or the mother or any maternal relative, since archival tumor tissue of the parents is not available. Another possibility is that the male breast case with the BRCA2 mutation might have a different father from the others. Furthermore, other male breast cancer patients, belonging to unrelated families, are negative for this specific mutation while they are positive for other recurrent BRCA2 mutations, such as 6174delT and 9326insA. The biopathological parameters of this BRCA2 cancer show an aggressive tumor phenotype and this is in contrast with the literature data characterizing other kinds of mutations. ${ }^{17}$

This study revealed the importance and the benefit deriving from the knowledge of an accurate family history in the context of genetic testing. In fact, the lack of information about the male breast cancer of individual $3: 27$, would have made it impossible to identify the second mutation and, moreover, it would have been misleading for the patient's daughter, negative for BRCA1 but resulting positive for BRCA2. The 3358T $\rightarrow$ A BRCA1 mutation was reported, for the first time, by Gayther et $a l^{18}$ in a family characterized by one ovarian and seven breast cancers. It was the only mutation, located in the first two-thirds of the coding sequence, where the breast cancer is higher than the ovarian cancer incidence. Further studies have shown that the frequency of ovarian cancer compared to breast cancer associated with mutations downstream to the granin motif (nucleotides 3760-3787) was significantly lower than for other mutations. ${ }^{19}$ Our data are in agreement with those of Thompson et $a l^{20}$ that, evaluating the genotype-phenotype correlations in 356 families with BrCa1 mutations, demonstrated that the mutations occurring in the regions $2401-4190$ are more frequently involved in the development of ovarian cancer rather than those mapping at nucleotides 1-2400.

Our findings suggest that the BRCA1 3358T $\rightarrow$ A mutation predisposes also to a high incidence of ovarian cancer with the consequence that the surveillance strategies must be different and, particularly, we believe appropriate to consider a prophylactic oophorectomy for the carrier. Although our data show that there is an elevated cancer risk for this family, the results do not allow us to draw conclusions on tumor types other than breast and ovarian cancer.

\section{Acknowledgements}

This work was supported by AIRC (Associazione Italiana per la Ricerca sul Cancro), COFIN-MURST 2001-2003 and Fondazione Cassa di Risparmio di Modena. 


\section{References}

1 Ramus SJ, Friedman LS, Gayther SA et al: A breast/ovarian cancer patient with germline mutations in both BRCA1 and BRCA2 [letter]. Genet 1997; 15: 14-15.

2 Liede A, Metcalfe K, Offit $\mathrm{K}$ et al: A family with three germline mutations in BRCA1 and BRCA2. Clin Genet 1998; 54: 215-218.

3 Tesoriero A, Andersen C, Southey $\mathrm{M}$ et al: De novo BRCA1 mutation in a patient with breast cancer and an inherited BRCA2 mutation [letter]. Am J Hum Genet 1999; 65: 567-569.

4 Gershoni-Baruch R, Dagan E, Kepten I, Freid G: Co segregation of BRCA1 185delAG mutation and BRCA2 6174delT in one single family. Eur J Cancer 1997; 33: 2283-2284.

5 Friedman E, Bar Sade Bruchim R, Kruglikova A et al: Double heterozygotes for the Ashkenazi founder mutations in BRCA1 and BRCA2 genes. Am J Hum Genet 1998; 63: 1224-1227.

6 Moslehi R, Russo D, Phelan C, Jack E, Antman K, Narod S: An unaffected individual from a breast/ovarian cancer family with germline mutations in both BRCA1 and BRCA2. Clin Genet 2000; 57: 70-73.

7 Ganguly A, Citron M, Godmilow L, Ahrens M, Ganguly T: Caucasian family with two independent mutations 2594delC in BRCA1 and 5392delAG in BRCA2. Am J Med Genet 2001; 101: 146-152.

8 Stoppa Lyonnet D, Fricker JP, Essioux L et al: Segregation of two BRCA1 in a single family. Am J Med Genet 1996; 59: $479-481$.

9 Liede A, Rehal P, Vesprini D, Jack E, Abrahamson J, Narod SA: A breast cancer patient of Scottish descent with germ line mutations in BRCA1 and BRCA2. Am J Hum Genet 1998; 62: 1543-1544.

10 Ludwig T, Fisher P, Ganesan S, Efstratiadis A: Tumorigenesis in mice carrying a truncating Brca1 mutation. Genes Dev 2001; 15: $1188-1193$.

11 Friedman LS, Ostermeyer EA, Szabo CI et al: Confirmation of BRCA1 by analysis of germline mutations linked to breast and ovarian cancer in ten families. Nat Genet 1994; 8: 399-404.

12 Cortesi L, Turchetti D, Bertoni C et al: Comparison between genotype and phenotype identifies a high risk population carrying BRCA1 mutations. Genes Chromosomes Cancer 2000; 27: 130-135.

13 Federico M: Registro tumori della provincia di Modena; in Zanetti R, Gafà L, Pannelli T, Conti R, Rosso S (Eds): Cancer in Italy: incidence data from cancer registries. Il Pensiero Scientifico Editore, Roma, 2002; Vol. III, pp. 309-319.

14 Couch FJ, Farid LM, DeShano ML et al: BRCA2 germline mutations in male breast cancer cases and breast cancer families. Nat Genet 1996; 13: 123-125.

15 Berchuck A, Heron KA, Carney ME et al: Frequency of germline and somatic BRCA1 mutations in ovarian cancer. Clin Cancer Res 1998; 4: 2433-2437.

16 Thompson D, Easton D, Breast Cancer Linkage Consortium: Variation in BRCA1 cancer risks by mutation position. Cancer Epidemiol Biomarkers Prev 2002; 11: 329-336.

17 Verhoog LC, Brekelmans CT, Seynaeve C et al: Survival in hereditary breast cancer associated with germline mutations of BRCA2. J Clin Oncol 1999; 17: 3396-3402.

18 Gayther SA, Warren W, Mazoyer S et al: Germline mutations of the BRCA1 gene in breast and ovarian cancer families provide evidence for a genotype-phenotype correlation. Nat Genet 1995; 11: 428-433.

19 Holt JT, Thompson ME, Szabo C et al: Growth retardation and tumour inhibition by BRCA1. Nat Genet 1996; 12: 298-302.

20 Thompson D, Easton D, Breast Cancer Linkage Consortium: Cancer incidence in BRCA1 mutation carriers. J Natt Cancer Inst 2002; 94: 1358-1365. 